\title{
Therapeutic outcome of early-phase clinical trials in multiple myeloma: a meta-analysis
}

\author{
Niels van Nieuwenhuijzen ${ }^{1,2}$, Rowan Frunt $\mathbb{D}^{1}$, Anne M. May ${ }^{3}$ and Monique C. Minnema (D)
}

\begin{abstract}
Great progress in the treatment of patients with multiple myeloma (MM) has been made due to the development of novel drugs. Patients with relapsed/refractory MM (RRMM) can be enrolled in early-phase clinical trials, but their performance across the last decade is unknown. We conducted a meta-analysis on the overall response rate (ORR) and toxicity. PubMed, Embase, and Cochrane Library were systematically searched for phase I and phase II trials investigating an experimental compound as a single agent or in combination with dexamethasone, published from January 1, 2010 to July 1, 2020. Eighty-eight articles were included, describing 61 phase I trials involving 1835 patients and 37 phase II trials involving 2644 patients. There was a high degree of heterogeneity. Using a random-effects model, the $95 \% \mathrm{Cls}$ of the estimated ORR were $8-17 \%$ for phase I trials and $18-28 \%$ for phase II trials. There were significant subgroup differences in ORR between the years of publication in phase I trials and between drug classes in both phase I and phase II trials. The ORR in early-phase clinical trials in RRMM is substantial, especially in phase II trials, but due to high heterogeneity a general assessment of clinical benefit before participation is difficult to offer to patients.
\end{abstract}

\section{Introduction}

Early-phase clinical trials in oncology are used to determine the safety profile, dosing regimen, and preliminary efficacy of an experimental drug. Phase I trials often represent the first-in-human use and are primarily used to assess toxicities, dosing schedule, and maximum tolerated dose (MTD). Historically, there was no or only very limited therapeutic intent in phase I trials. Phase II trials test the selected dosing regimen in a larger sample to acquire insight into the effectiveness of experimental drugs. In oncology, participants in early-phase clinical trials typically have the advanced-stage disease and have exhausted one or more lines of approved therapeutic regimens. Participants are to expect a higher risk of

\footnotetext{
Correspondence: Monique C. Minnema (m.c.minnema@umcutrecht.nl) 'Department of Hematology, University Medical Center Utrecht, Utrecht University, Utrecht, The Netherlands

${ }^{2}$ Center for Translational Immunology, University Medical Center Utrecht, Utrecht University, Utrecht, The Netherlands

Full list of author information is available at the end of the article
}

toxicities, while the activity of the experimental drug is uncertain $^{1,2}$.

In the 1970s and 1980s, overall response rates (ORR) in phase I trials in oncology were estimated to be $<5 \%^{3-5}$. However, rates of therapeutic success have improved since, with estimated responses of $5-10 \%$ in the 1990 s and estimates for the past two decades varying from 5 to $20 \%{ }^{6-9}$. The American Society of Clinical Oncology, among others, perceives phase I trials as having therapeutic intent and sees participation as a therapeutic option $^{1,2}$. However, it remains subject to debate whether phase I trials genuinely offer therapeutic benefit in relation to their risks ${ }^{1,2,10-12}$.

Multiple myeloma (MM) is a clonal plasma cell malignancy primarily located in the bone marrow and is the second most common hematologic malignancy ${ }^{13}$. There have been many drug approvals for MM in the last 15 years, which have significantly prolonged survival for patients ${ }^{14}$. Despite significant improvements in treatment possibilities and survival, MM remains an incurable malignancy and is characterized by high rates of relapse 
and therapy resistance $e^{13,15,16}$. In the course of disease progression, participation in an early-phase clinical trial can be considered. However, a recent systematic review to inform clinicians and patients on the risks and benefits of these trials is lacking. Therefore, we examined the rates of response and toxicities in early-phase clinical trials in relapsed/refractory MM (RRMM) and assessed differences across years of publication and drug classes.

\section{Methods}

\section{Search strategy and study selection}

This systematic review is reported in accordance with the Preferred Reporting Items for Systematic Reviews and Meta-Analyses (PRISMA) guidelines ${ }^{17}$. Two researchers (NvN and RF) independently searched the publication libraries of PubMed, EMBASE, and the Cochrane Central Register of Controlled Trials from January 1, 2010 to July 1 , 2020. Search strings were formulated and verified in accordance with a Utrecht University librarian. The complete search strings are provided in the Supplementary Materials. Searches were limited to publications in the English language. We identified all publications reporting on single-arm phase I and phase II clinical trials with adult RRMM patients that received an experimental compound given as monotherapy or in combination with dexamethasone. Additional relevant articles that were found through other sources were reviewed similarly and could be included when eligibility criteria were met. Studies, where the experimental compound was combined with drugs, other than dexamethasone, were excluded. Studies were also excluded when testing the effect of a drug in a subgroup of RRMM patients, e.g., safety studies in patients with renal failure, or when studies were performed for the mere purpose of approval in one specific country. We further excluded studies related to allogeneic stem cell therapy, bone-directed therapy or supportive care, or studies testing a new route of administration. Studies had to report the activity of the experimental compound on an intention-to-treat population of at least ten RRMM patients.

\section{Data extraction}

Results were extracted from the included studies and tabulated by two researchers ( $\mathrm{NvN}$ and $\mathrm{RF}$ ) independently. Potential conflicts were resolved by discussion with a third researcher (MCM). In case of missing data, corresponding authors of the studies were contacted. When we identified multiple publications on the same study population, results from the most recent and/or most complete original publication were used for data extraction. When not all data could be extracted from the original article, a trial registration database (ClinicalTrials. gov) was used to find additional relevant publications in order to obtain missing data. For studies that reported the results of a combined phase I and phase II trial, we manually extracted data of patients belonging to the respective trial phase.

The primary outcome was the ORR, defined as a bestreported partial response (PR) or better. Secondary outcomes were toxicities, measured as dose-limiting toxicities (DLTs) and drug discontinuations, and the clinical benefit rate (CBR): a best-reported response of minimal response (MR) or better ${ }^{18}$. We included CBR since also a minor response can be a signal of single-drug activity and may have additional value to RRMM patients ${ }^{19}$. From each included study, we extracted the following characteristics: trial stage, Clinical Trial Registration Number, name, type, and a class of experimental drug, intentionto-treat population, median lines of prior therapy, response rates: ORR $(\geq \mathrm{PR})$ and $\mathrm{CBR}(\geq \mathrm{MR})$, toxicity profile: number of DLTs or number of patients discontinuing the trial drug due to adverse events. Wherever possible, we extracted the reported response rates according to the International Myeloma Working Group definitions ${ }^{18}$.

\section{Statistical analysis}

We used statistical software R, version 4.0.0, for all data analyses $^{20}$. The package "meta" (4.12-0) was used for meta-analyses ${ }^{21}$. We applied logit transformations to turn the proportions into normal distributions suitable to pool effects. The weight of each included study in the metaanalysis was determined with the generic inverse variance method. This means that larger studies, which generally have smaller variances than small studies, will be given a relatively greater weight within the meta-analysis. Effect sizes from each study were then pooled with a randomeffects model. Preplanned subgroup analyses were devised for years of publication and drug class. To evaluate the degree of statistical heterogeneity, we used the Cochran Q statistic and inconsistency index $\left(I^{2}\right)$. Heterogeneity was classified as low $\left(I^{2}=25 \%\right)$, moderate $\left(I^{2}=50 \%\right)$, and high $\left(I^{2}=75 \%\right)^{22}$. All effect sizes are reported with corresponding 95\% confidence intervals (CI) and prediction intervals (PI). Reported $P$ values are two-sided, with a $P<$ 0.05 considered significant. We used the test for subgroup differences to investigate how the estimates vary across subgroups of years of publication and drug classes.

\section{Results}

\section{Selection of studies}

Our literature search identified 1127 original publications for review after the removal of duplicates. A further eight articles were found through other sources and added to the selection, making the total 1135 publications. After screening on title and abstract, 110 potentially relevant articles were selected for full-article review on eligibility. Of these, 88 studies were included for data extraction and 


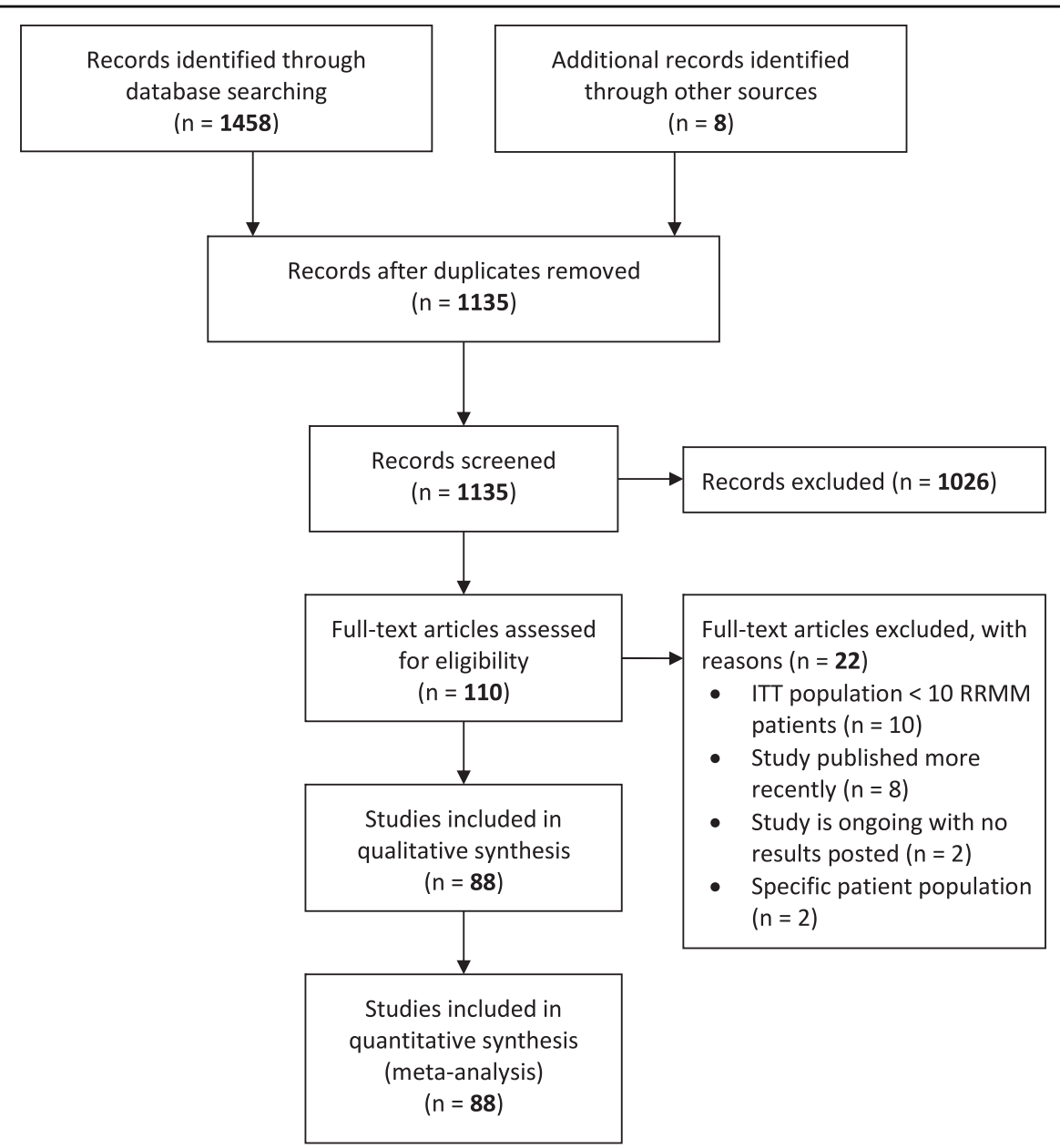

Fig. 1 PRISMA flow diagram of the study selection process. ITT intention-to-treat, RRMM relapsed/refractory multiple myeloma.

quantitative analysis (Supplementary eTable 1$)^{23-112}$. The selection process is visualized in Fig. 1.

\section{Study and patient characteristics}

The 88 studies that were included for analysis together report the results of 98 clinical trials, of which 61 phase I trials and 37 phase II trials. Several publications reported the results of both a phase I and phase II trial. In total, 4479 patients were included, of which 1835 in phase I trials and 2644 in phase II trials (Supplementary eTable 2).

We divided all included studies into four subgroups based on their year of publication: 2010-2012 $(n=16)$, 2013-2015 $(n=22), 2016-2018(n=35)$, and 2019-2020 $(n=26)$ (Table 1$)$. In addition, we subdivided the studies into nine different subgroups based on the drug class of the experimental compound: immunomodulatory imide drugs (IMiDs) $(n=4)$, proteasome inhibitors $(n=20)$, monoclonal antibodies $(n=18)$, cell therapy $(n=5)$, antibody-drug conjugates $(n=7)$, kinase inhibitors $(n=10)$, immune checkpoint inhibitors $(n=2)$, heat- shock protein 90 inhibitors $(n=5)$, and others $(n=27)$. Thirty-five (58\%) of phase I trials and 29 (78\%) of phase II trials reported on the median lines of prior therapies received by patients before participation in the trial. Median lines of prior therapies ranged from two to more than seven, while patients in phase I trials seem to have been more heavily pretreated than patients in phase II trials (Table 1). In 39 (40\%) trials, dexamethasone was given in addition to the novel compound from the start of the trial. Most trials were designed specifically for RRMM; five trials were set up to test the experimental drug in other malignancies as well.

\section{Response rate}

The ORR to the experimental drug was reported in all but one of the included trials. The degree of heterogeneity between the studies for ORR was high in both phase I $\left(I^{2}=84 \%, P<0.001\right)$ and phase II studies $\left(I^{2}=84 \%\right.$, $P<0.001)$. The $95 \% \mathrm{CI}$ of the estimated overall response was $8.1-16.8 \%$ for all included phase I trials and 
Table 1 Included studies per subgroup.

\begin{tabular}{|c|c|c|c|}
\hline & $\begin{array}{l}\text { Phase I, } \\
N=61 \\
\text { No. (\%) }\end{array}$ & $\begin{array}{l}\text { Phase II, } \\
N=37 \\
\text { No. (\%) }\end{array}$ & $\begin{array}{l}\text { Total, } \\
N=98 \\
\text { No. (\%) }\end{array}$ \\
\hline \multicolumn{4}{|l|}{ Years } \\
\hline 2010-2012 & $9(15)$ & $7(19)$ & $16(16)$ \\
\hline 2013-2015 & $14(23)$ & $8(22)$ & $22(22)$ \\
\hline 2016-2018 & $22(36)$ & $13(35)$ & $35(36)$ \\
\hline 2019-2020 & $16(26)$ & $9(24)$ & $25(26)$ \\
\hline \multicolumn{4}{|l|}{ Drug } \\
\hline IMiD & $1(2)$ & $3(8)$ & $4(4)$ \\
\hline PI & $9(15)$ & $11(30)$ & $20(20)$ \\
\hline $\mathrm{mAb}$ & $12(20)$ & $6(16)$ & $18(18)$ \\
\hline Cell therapy & $6(10)$ & - & $6(6)$ \\
\hline$A D C$ & $6(10)$ & $1(3)$ & $7(7)$ \\
\hline Kinase inhibitor & $5(8)$ & $5(14)$ & $10(10)$ \\
\hline $\mathrm{ICl}$ & $2(3)$ & - & $2(2)$ \\
\hline Hsp90i & $5(8)$ & - & $5(5)$ \\
\hline Other & $15(25)$ & $11(30)$ & $26(27)$ \\
\hline \multicolumn{4}{|c|}{ Median lines of prior therapy } \\
\hline $1-3$ & $7(11)$ & $9(24)$ & $16(16)$ \\
\hline $4-6$ & $22(36)$ & $18(49)$ & $40(41))$ \\
\hline$\geq 7$ & $7(11)$ & $2(5)$ & $9(9)$ \\
\hline Unknown & $25(41)$ & $8(22)$ & $33(34)$ \\
\hline
\end{tabular}

IMiD immunomodulatory imide drugs, PI proteasome inhibitors, $m A b$ monoclonal antibodies, $A D C$ antibody-drug conjugate, $\mathrm{ICl}$ immune checkpoint inhibitor, Hsp90i heat-shock protein 90-inhibitor.

18.9-28.3\% for all included phase II trials. The prediction interval of the estimated overall responses was $0.8-67.6 \%$ for phase I trials and $6.9-55.2 \%$ for phase II trials (Figs. 2 and 3).

We examined possible associations between the estimated response and subgroups of trials based on the year of publication. Subgroup analysis revealed a significant difference between years of publication in phase I trials $(P=0.01)$, but not in phase II trials $(P=0.07)$ (Fig. 4$)$. In phase I trials, the $95 \% \mathrm{CI}$ of the response estimate of trials published from January 1, 2010 to December 31, 2012 was $1.7-9.7 \%$, while for trials published from January 1 , 2019 to July 1, 2020 the 95\% CI was $12.4-37.3 \%$. Furthermore, ORR estimates varied according to the drug class of the investigational compound. The test for subgroup differences demonstrated a significant difference between drug classes for both phase I $(P<0.0001)$ and phase II $(P<0.0001)$ trials (Fig. 4$)$. In phase I trials, the 95\% CI of the response estimates were low for drug classes Hsp90i (0.7-7.7\%) and kinase inhibitors
(2.9-13.7\%), and high for drug classes PI (15.6-44.5\%) and cell therapy (44.7-85.8\%). Since most of the CAR Tcell trials were published in 2019, these trials seem to be responsible for the association between ORR and the year of publications (Supplementary eTable 3$)^{113,114}$.

\section{Toxicities}

The rate of DLTs in phase I trials and rate of drug discontinuations in phase II trials were collected as surrogate markers of important drug-related toxicities. We were able to gather the number of DLTs for 51 of 60 phase I trials and the number of drug discontinuations in 29 out of 37 clinical trials. For phase I trials, there was an intermediate degree of heterogeneity $\left(I^{2}=31.5 \%, P=\right.$ $0.02)$. The estimated proportion of DLTs was $9.1 \%(95 \%$ CI $7.3-11.4 \%$ ). The prediction interval was $3.6-21.3 \%$. (Fig. 2). For phase II trials, heterogeneity was scored as borderline intermediate $\left(I^{2}=74.6 \%, P<0.001\right)$. The estimated overall number of drug discontinuations was $12.49 \%$ (95\% CI 9.7-15.9\%), with the prediction interval ranging from 3.7 to $34.4 \%$ (Fig. 3).

For the estimate of DLTs in phase I trials, we did not find any subgroup differences for both years of publication $(P=0.77)$ and drug class $(P=0.10)$. For drug discontinuations in phase II trials, there were significant subgroup differences for drug class $(P=0.03)$ but not for years of publication $(P=0.55)$.

\section{Clinical benefit rate}

The CBR was reported in 48 of 60 phase I trials and 30 of 37 phase II trials. Again, heterogeneity between individual studies was high for both phase I $\left(I^{2}=79 \%, P<\right.$ $0.001)$ and phase II trials $\left(I^{2}=86 \%, P<0.01\right)$. The $95 \% \mathrm{CI}$ of the estimated overall CBR was $13.7-24.1 \%$ for phase I trials, including 1456 patients, and $28.7-40.4 \%$ for phase II trials, involving 2397 patients. The prediction interval of the estimated overall CBR was 3.0-62.4\% for phase I trials and $12.4-65.8 \%$ for phase II trials (Supplementary eFig. 1).

Similar to the ORR, we analyzed subgroup differences across different years of publication and different drug classes. The test for subgroup differences was significant for years of publication for phase I $(P=0.01)$ but not phase II $(P=0.2)$ trials. In addition, there was a significant difference between drug classes for both phase I $(P<$ $0.001)$ and phase II $(P<0.011)$ studies.

\section{Discussion}

Patients with RRMM will inevitably exhaust multiple lines of approved treatment regimens since it currently remains an incurable disease. In specific trial centers, fit patients are offered enrollment in early-phase clinical trials, but contemporary data on the performance and toxicities of these types of trials is lacking for $\mathrm{MM}^{1,2,10}$. In this systematic review and meta-analysis of early-phase 


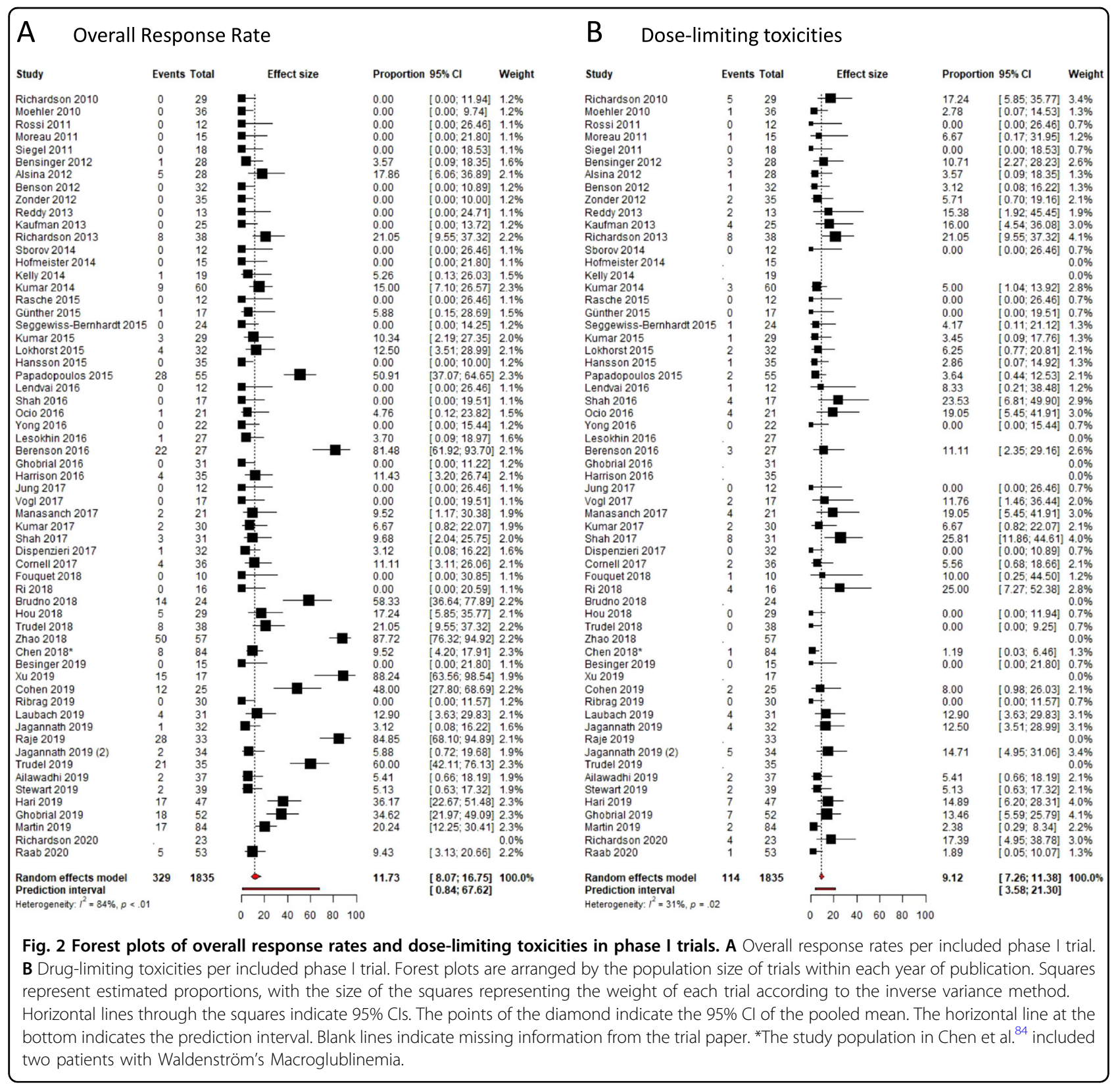

clinical trials, we assessed the potential benefit and risks to patients across the past decade.

We searched for trials that investigate single-drug activity in RRMM because these types of drugs are most likely to be beneficial to patients in follow-up randomized phase III studies. Since dexamethasone is so frequently used, also to prevent side effects of the experimental drug, we did allow for its use in the current analysis. In total, we found 88 original studies, which is a considerable number of studies for a meta-analysis of early-phase clinical trials in one specific disease $e^{6-8}$.

The heterogeneity between the individual studies was high, with $I^{2}$ consistently above $75 \%$. This was to be expected with the inclusion of trials testing therapy with widely varying modes of action. Therefore, we considered it inappropriate to provide a single estimate of the response rates as a mean of all early-phase trials. Instead, we provided the $95 \%$ CI of the estimated mean and the prediction intervals. The $95 \%$ CI presents a summary of the treatment effect, while the prediction interval shows the range of effects that can be expected in future similar studies. The prediction interval implies that the ORR of a future phase I trial may range from $0.8 \%$ all the way up to $67.6 \%$, based on results from the past decade (Fig. 2). The $95 \%$ CI of the estimated response rate of phase I trials was $8.1-16.8 \%$, which is comparable to recent analyses on 


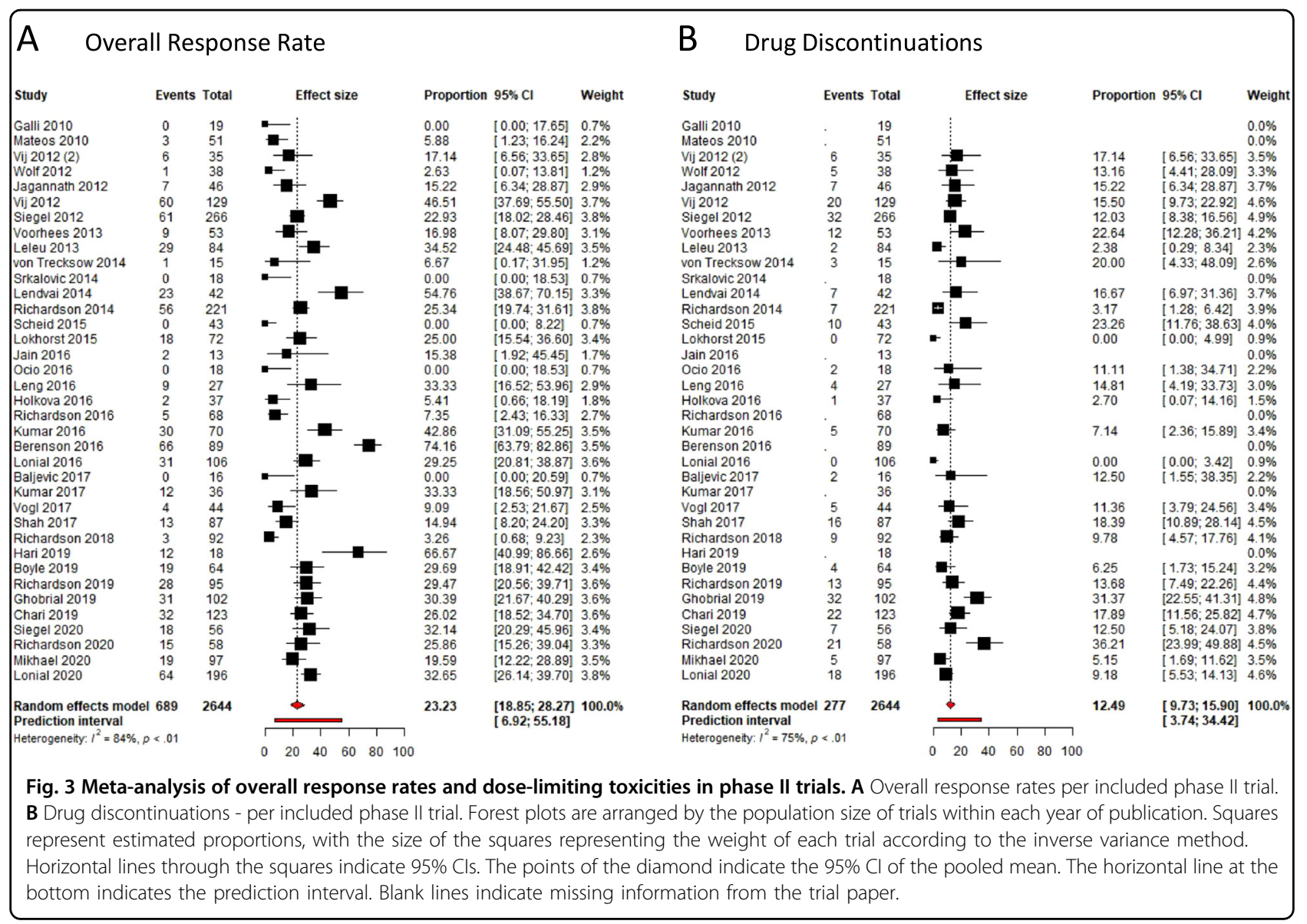

therapeutic effectivity of phase I trials in oncology for non-hematological malignancies ${ }^{8,9}$.

Our results show that response rates are dependent on the drug class of the experimental compound (Figs. 2 and 4). Response rates of approved and currently widely used drugs such as PI or IMiDs are better than drugs such as Hsp90-inhibitors or kinase inhibitors. The latter has failed to demonstrate activity as a single agent and have not progressed to phase III trials. Notably, anti-B Cell Maturation Antigen (BCMA) CAR T cells have shown remarkable activity in relapsed MM patients with a $95 \%$ CI of $44.5-85.8 \%^{113,114}$. However, performing the metaanalysis without these trials only reduced heterogeneity by several percentage points (Supplementary eTable 3). Currently, results have only been published for phase I studies of anti-BCMA CAR $\mathrm{T}$ cells, but phase II results are expected with great anticipation.

Patients with active RRMM can also benefit from a treatment that merely prevents further disease progression and thereby avoids the development of myelomarelated symptoms. Therefore, we analyzed the CBR of the included trials. A MR indicates that the drug used as monotherapy has some activity in the disease under investigation and may be suitable for combination strategies in the future. Effect estimates of the CBR (phase I: 13.7-24.1\%; phase II: 28.7-40.4\%) were higher than for the ORR (phase I: $8.1-16.8 \%$; phase II: $18.9-28.3 \%$ ), while the range of the prediction interval of the CBR was comparable to the prediction interval of the ORR (Fig. 2 and Supplementary eFig. 1).

As a surrogate marker for important drug-related toxicities, we chose the rate of DLTs in phase I trials and drug discontinuations in phase II trials. DLTs are standardly described in phase I trial protocols and are used to establish the MTD of the experimental drug. There was a consistent rate of DLTs in phase I studies of $9.1 \%$ and a rate of drug discontinuations of $12.5 \%$ in phase II studies (Fig. 2). With the advent of targeted therapies and immune therapies, the linear relationship between dose and efficacy is less apparent and the same holds true for the relationship between dose and toxicities.

The trials that are pooled are heterogeneous due to the different interventions used. A major limitation of our study is the considerable heterogeneity that exists between individual trials as a result of the prior drug exposure of patients. This is reflected by differences in the median line of therapies received before participating in a trial, but the response to the experimental drug also 


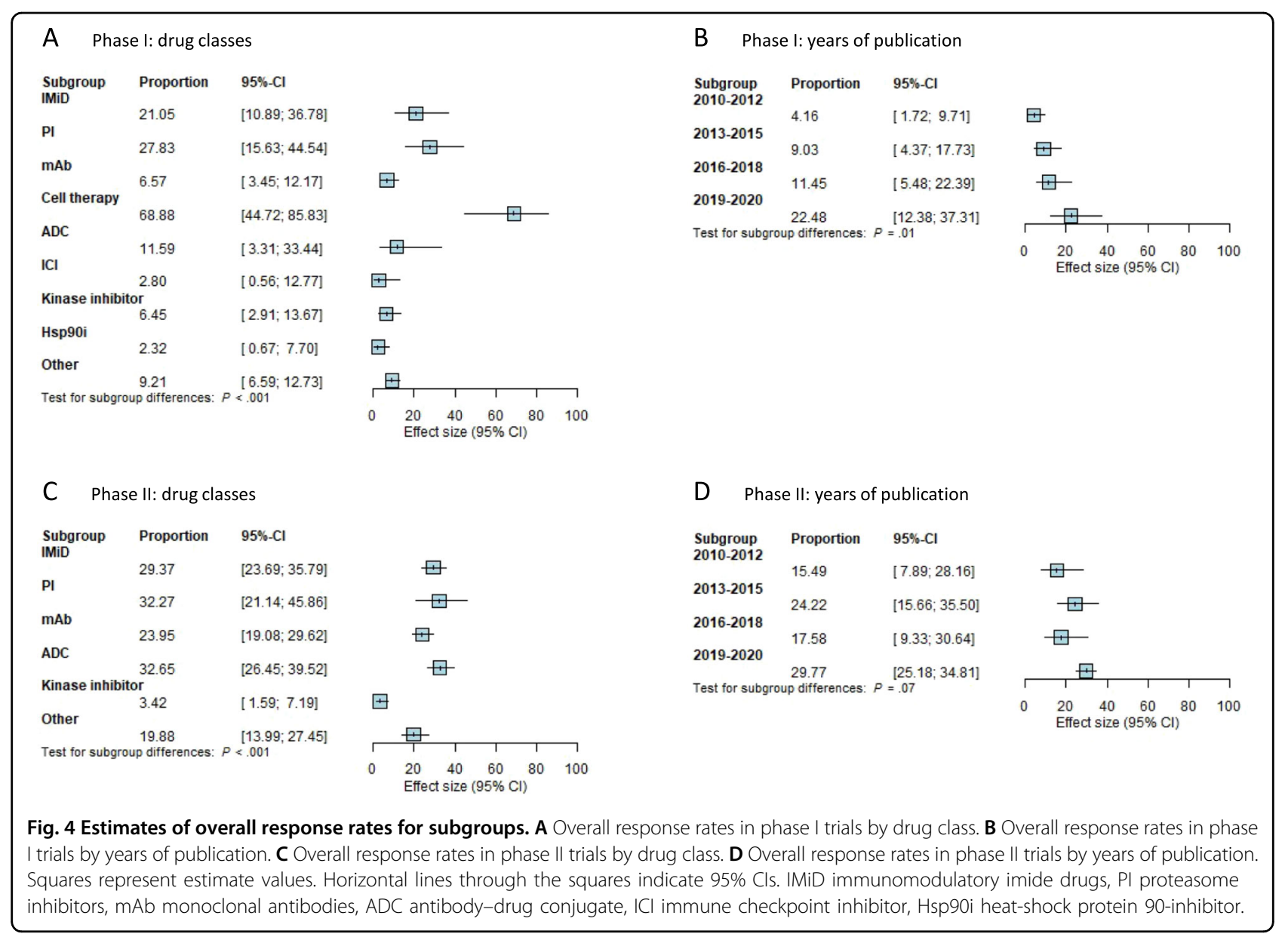

depends on the type of drugs a patient has received at initial diagnosis and subsequent relapses. Therapy regimens have not only changed considerably over the past decade but are also dependent on what region of the world the patient is treated in. Therefore, trial results are dependent on how, when, and where a patient was treated previously. As such the heterogeneity in patient populations between trials poses limitations on the generalizability of our findings. Another shortcoming of earlyphase clinical trials is that the primary outcome is the best response, which is a surrogate marker for improved overall survival (OS). Although ORR and OS are associated, a higher ORR might not necessarily implicate an improvement in $\mathrm{OS}^{115}$. Progression-free survival and OS are better measurements of clinical benefit but are not generally reported for early-phase clinical trials.

There are several factors that may contribute to a further increase in the efficacy of early-phase clinical trials in the near future. Part of the heterogeneity between studies was due to the high ORR of studies testing anti-BCMA CAR T cells ${ }^{114}$. These and other promising novel forms of immune therapy may substantially increase response rates without increasing toxicity. Another step that may improve therapeutic success is an intrapatient dose escalation, which helps patients to receive safe and higher doses of a potentially active novel drug. In addition, biomarker selection can improve the selection process of patients likely to benefit from a novel drug ${ }^{116}$.

\section{Conclusions}

The increased understanding of cancer pathobiologyand MM especially-has resulted in many novel drugs that specifically target malignant cells. Immunotherapies have been developed for MM patients and have opened a completely new set of treatment possibilities. Consequently, response rates in early-phase clinical trials have improved over the past decades. In this meta-analysis, we characterized the overall effect of participating in earlyphase clinical trials for RRMM patients over the past decade. We found that the ORR of early-phase clinical trials is highly variable, but seems to have improved over the past decade without a concurrent rise in toxicities. These improvements are mainly due to novel classes of therapy, such as PIs, IMiDs, and CAR T cells. Our findings can be used to facilitate debate on the risks and benefits of participation in early-phase clinical trials in 
oncology in general and may be used by clinicians to guide assessment and communication on enrollment of RRMM patients in early-phase clinical trials.

\section{Acknowledgements}

There was no funding source for this study.

\section{Author details}

'Department of Hematology, University Medical Center Utrecht, Utrecht University, Utrecht, The Netherlands. ${ }^{2}$ Center for Translational Immunology, University Medical Center Utrecht, Utrecht University, Utrecht, The Netherlands. ${ }^{3}$ Julius Center for Health Sciences and Primary Care, University Medical Center Utrecht, Utrecht University, Utrecht, The Netherlands

\section{Conflict of interest}

Prof. Dr. M.C.M. declares the following potential conflicts of interest: Research funding: Celgene (paid to the employer). Advisory Boards: Celgene, Jansen Cilag, Amgen, Takeda, Servier (paid to the employer). Training: Gilead (paid to the employer). The remaining authors declare no competing interests.

\section{Publisher's note}

Springer Nature remains neutral with regard to jurisdictional claims in published maps and institutional affiliations.

Supplementary information The online version contains supplementary material available at https://doi.org/10.1038/s41408-021-00441-3.

Received: 11 December 2020 Revised: 3 February 2021 Accepted: 10 February 2021

Published online: 01 March 2021

\section{References}

1. Weber, J. S. et al. American society of clinical oncology policy statement update: the critical role of phase I trials in cancer research and treatment. $J$. Clin. Oncol. 33, 278-284 (2015).

2. Adashek, J. J., LoRusso, P. M., Hong, D. S. \& Kurzrock, R. Phase I trials as valid therapeutic options for patients with cancer. Nat. Rev. Clin. Oncol. 16, 773-778 (2019).

3. Estey, E. et al. Therapeutic response in phase I trials of antineoplastic agents. Cancer Treat. Rep. 70, 1105-1113 (1986).

4. Decoster, G., Stein, G. \& Holdener, E. E. Responses and Toxic Deaths in Phase Clinical Trials, Vol I (Khtwer Academic Publishers, 1990).

5. Itoh, K. et al. Therapeutic response and potential pitfalls in phase I clinical trials of anticancer agents conducted in Japan. Cancer Chemother. Pharmacol. 34, 451-454 (1994)

6. Horstmann, E. et al. Risks and benefits of phase 1 oncology trials, 1991 through 2002. N. Engl. J. Med. 352, 895-904 (2005).

7. Roberts, T. G. et al. Trends in the risk and benefits to patients with cancer participating in phase 1 clinical trials. J. Am. Med. Assoc. 292, 2130-2140 (2004).

8. Fukuda, Y. K. et al. Risks and benefits of phase 1 oncology trials, 2001 through 2012. J. Clin. Oncol. 32, 2552-2552 (2014).

9. Chakiba, C., Grellety, T., Bellera, C. \& Italiano, A. Encouraging trends in modern phase 1 oncology trials. N. Engl. J. Med. 378, 2242-2243 (2018).

10. Kimmelman, J. Phase I trials as therapeutic options: (usually) a betrayal of evidence-based medicine. Nat. Rev. Clin. Oncol. 16, 719-720 (2019).

11. Pentz, R. D. et al. Therapeutic misconception, misestimation, and optimism in participants enrolled in phase 1 trials. Cancer 118, 4571-4578 (2012).

12. Tao, D. L., Kartika, T., Tran, A. \& Prasad, V. Phase I trials and therapeutic intent in the age of precision oncology: what is a patient's chance of response? Eur. J. Cancer 139, 20-26 (2020)

13. Kumar, S. K. et al. Multiple myeloma. Nat. Rev. Dis. Prim. 3, 1-20 (2017).

14. Thorsteinsdottir, S. et al. Dramatically improved survival in multiple myeloma patients in the recent decade: results from a Swedish population-based study. Haematologica 103, e412-e415 (2018)
15. Laubach, J. et al. Management of relapsed multiple myeloma: recommendations of the International Myeloma Working Group. Leukemia 30, 1005-1017 (2016).

16. Kumar, S. K. et al. Natural history of relapsed myeloma, refractory to immunomodulatory drugs and proteasome inhibitors: a multicenter IMWG study. Leukemia 31, 2443-2448 (2017).

17. Moher, D., Liberati, A., Tetzlaff, J. \& Altman, D. G. Preferred reporting items for systematic reviews and meta-analyses: the PRISMA statement. PLoS Med. $\mathbf{6}$, e1000097 (2009).

18. Kumar, S. et al. International Myeloma Working Group consensus criteria for response and minimal residual disease assessment in multiple myeloma. Lancet Oncol. 17, e328-e346 (2016).

19. Chan, E. H. L. et al. Clinical benefit of depth of response for relapsed/ refractory multiple myeloma patients treated on clinical trials: retrospective analysis from two tertiary centres. Br. J. Haematol. 186, 162-165 (2019).

20. R Core Team. R: A language and environment for statistical computing. R Foundation for Statistical Computing, Vienna, Austria; https://www.R-project. org/ (2020).

21. Balduzzi, S., Rücker, G. \& Schwarzer, G. How to perform a meta-analysis with R: a practical tutorial. Evidence-Based Mental Health 22, 153-160 (2019).

22. Higgins, J. P. T., Thompson, S. G., Deeks, J. J. \& Altman, D. G. Measuring inconsistency in meta-analyses. Br. Med. J. 327, 557-560 (2003).

23. Richardson, P. G. et al. Tanespimycin monotherapy in relapsed multiple myeloma: results of a phase 1 dose-escalation study. Br. J. Haematol. 150, 438-445 (2010).

24. Moehler, T. M. et al. Combined phase I/II study of imexon (AOP99.0001) for treatment of relapsed or refractory multiple myeloma. Anticancer Drugs 21, 708-715 (2010).

25. Mateos, M. V. et al. Phase II clinical and pharmacokinetic study of plitidepsin 3-hour infusion every two weeks alone or with dexamethasone in relapsed and refractory multiple myeloma. Clin. Cancer Res. 16, 3260-3269 (2010).

26. Galli, M. et al. A phase II multiple dose clinical trial of histone deacetylase inhibitor ITF2357 in patients with relapsed or progressive multiple myeloma. Ann. Hematol. 89, 185-190 (2010).

27. Rossi, J.-F. Phase I study of atacicept in relapsed/refractory multiple myeloma (MM) and Waldenström's macroglobulinemia. Clin. Lymphoma Myeloma Leuk. 11, 136-138 (2011)

28. Moreau, P. et al. Phase I study of the anti insulin-like growth factor 1 receptor (IGF-1R) monoclonal antibody, AVE1642, as single agent and in combination with bortezomib in patients with relapsed multiple myeloma. Leukemia $\mathbf{2 5}$ 872-874 (2011).

29. Siegel, D. et al. A phase 1 study of IPI-504 (retaspimycin hydrochloride) in patients with relapsed or relapsed and refractory multiple myeloma. Leuk Lymphoma 52, 2308-2315 (2011).

30. Wolf, J. L. et al. Phase II trial of the pan-deacetylase inhibitor panobinostat as a single agent in advanced relapsed/refractory multiple myeloma. Leuk. Lymphoma 53, 1820-1823 (2012).

31. Vij, R. et al. An open-label, single-arm, phase 2 (PX-171-004) study of singleagent carfilzomib in bortezomib-naive patients with relapsed and/or refractory multiple myeloma. Blood 119, 5661-5670 (2012).

32. Zonder, J. A. et al. A phase 1, multicenter, open-label, dose escalation study of elotuzumab in patients with advanced multiple myeloma. Blood $\mathbf{1 2 0}$ 552-559 (2012).

33. Vij, R. et al. An open-label, single-arm, phase 2 study of single-agent carfilzomib in patients with relapsed and/or refractory multiple myeloma who have been previously treated with bortezomib. Br. J. Haematol. 158, 739-748 (2012).

34. Alsina, M. et al. A phase I single-agent study of twice-weekly consecutive-day dosing of the proteasome inhibitor carfilzomib in patients with relapsed or refractory multiple myeloma or lymphoma. Clin. Cancer Res. 18, 4830-4840 (2012).

35. Bensinger, W. et al. A phase 1 study of lucatumumab, a fully human antiCD40 antagonist monoclonal antibody administered intravenously to patients with relapsed or refractory multiple myeloma. Br. J. Haematol. 159 58-66 (2012).

36. Jagannath, S. et al. An open-label single-arm pilot phase II study (PX-171-003A0) of low-dose, single-agent carfilzomib in patients with relapsed and refractory multiple myeloma. Clin. Lymphoma Myeloma Leuk. 12, 310-318 (2012). 
37. Siegel, D. S. et al. A phase 2 study of single-agent carfilzomib (PX-171-003-A1) in patients with relapsed and refractory multiple myeloma. Blood 120 2817-2825 (2012).

38. Benson, D. M. J. et al. A phase 1 trial of the anti-KIR antibody IPH2101 in patients with relapsed/refractory multiple myeloma. Blood 120, 4324-4333 (2012).

39. Leleu, X. et al. Pomalidomide plus low-dose dexamethasone is active and well tolerated in bortezomib and lenalidomide-refractory multiple myeloma: Intergroupe Francophone du Myélome 2009-02. Blood 121, 1968-1975 (2013).

40. Richardson, P. G. et al. Phase 1 study of pomalidomide MTD, safety, and efficacy in patients with refractory multiple myeloma who have received lenalidomide and bortezomib. Blood 121, 1961-1967 (2013).

41. Voorhees, P. M. et al. A phase 2 multicentre study of siltuximab, an antiinterleukin-6 monoclonal antibody, in patients with relapsed or refractory multiple myeloma. Br. J. Haematol. 161, 357-366 (2013).

42. Reddy, N. et al. Phase i trial of the HSP90 inhibitor PF-04929113 (SNX5422) in adult patients with recurrent, refractory hematologic malignancies. Clin. Lymphoma, Myeloma Leuk. 13, 385-391 (2013).

43. Kaufman, J. L. et al. Phase I, multicentre, dose-escalation trial of monotherapy with milatuzumab (humanized anti-CD74 monoclonal antibody) in relapsed or refractory multiple myeloma. Br. J. Haematol. 163, 478-486 (2013).

44. von Tresckow, B. et al. Anti-epidermal growth factor receptor antibody cetuximab in refractory or relapsed multiple myeloma: a phase II prospective clinical trial. Leuk. Lymphoma 55, 695-697 (2014).

45. Kelly, K. R. et al. Phase I study of MLN8237-investigational Aurora A kinase inhibitor-in relapsed/refractory multiple myeloma, non-Hodgkin lymphoma and chronic lymphocytic leukemia. Investig. New Drugs 32, 489-499 (2014).

46. Hofmeister, C. C. et al. A phase I trial of flavopiridol in relapsed multiple myeloma. Cancer Chemother. Pharmacol. 73, 249-257 (2014).

47. Richardson, P. G. et al. Pomalidomide alone or in combination with low-dose dexamethasone in relapsed and refractory multiple myeloma: a randomized phase 2 study. Blood 123, 1826-1832 (2014).

48. Lendvai, N. et al. A phase 2 single-center study of carfilzomib $56 \mathrm{mg} / \mathrm{m} 2$ with or without low-dose dexamethasone in relapsed multiple myeloma. Blood 124, 899-906 (2014).

49. Kumar, S. K. et al. Phase 1 study of weekly dosing with the investigational oral proteasome inhibitor ixazomib in relapsed/refractory multiple myeloma. Blood 124, 1047-1055 (2014).

50. Srkalovic, G. et al. A phase II trial of BAY 43-9006 (sorafenib) (NSC-724772) in patients with relapsing and resistant multiple myeloma: SWOG S0434. Cancer Med. 3, 1275-1283 (2014)

51. Sborov, D. W. et al. A phase I trial of single-agent reolysin in patients with relapsed multiple myeloma. Clin. Cancer Res. 20, 5946-5955 (2014).

52. Papadopoulos, K. P. et al. Phase I study of 30-minute infusion of carfilzomib as single agent or in combination with low-dose dexamethasone in patients with relapsed and/or refractory multiple myeloma. J. Clin. Oncol. 33, 732-739 (2015).

53. Seggewiss-Bernhardt, R. et al. Phase 1/1B trial of the heat shock protein 90 inhibitor NVP-AUY922 as monotherapy or in combination with bortezomib in patients with relapsed or refractory multiple myeloma. Cancer 121, 2185-2192 (2015).

54. Lokhorst, H. M. et al. Targeting CD38 with daratumumab monotherapy in multiple myeloma. N. Engl. J. Med. 373, 1207-1219 (2015).

55. Günther, A. et al. Activity of everolimus (RAD001) in relapsed and/or refractory multiple myeloma: a phase I study. Haematologica 100, 541-547 (2015).

56. Rasche, L. et al. GRP78-directed immunotherapy in relapsed or refractory multiple myeloma-results from a phase 1 trial with the monoclonal immunoglobulin M antibody PAT-SM6. Haematologica 100, 377-384 (2015).

57. Kumar, S. K. et al. Dinaciclib, a novel CDK inhibitor, demonstrates encouraging single-agent activity in patients with relapsed multiple myeloma. Blood 125, 443-448 (2015)

58. Hansson, M. et al. A phase I dose-escalation study of antibody Bl-505 in relapsed/refractory multiple myeloma. Clin. Cancer Res. 21, 2730-2736 (2015).

59. Scheid, C. et al. Phase 2 study of dovitinib in patients with relapsed or refractory multiple myeloma with or without $t(4 ; 14)$ translocation. Eur. J. Haematol. 95, 316-324 (2015).

60. Lonial, S. et al. Daratumumab monotherapy in patients with treatmentrefractory multiple myeloma (SIRIUS): an open-label, randomised, phase 2 trial. Lancet 387, 1551-1560 (2016).
61. Ocio, E. M. et al. Phase $\mathrm{I} / \mathrm{Il}$ study of weekly PM00104 (Zalypsis ${ }^{\circledR}$ ) in patients with relapsed/refractory multiple myeloma. Br. J. Haematol. 172, 625-628 (2016).

62. Ghobrial, I. M. et al. TAK-228 (formerly MLN0128), an investigational oral dual TORC1/2 inhibitor: a phase I dose escalation study in patients with relapsed or refractory multiple myeloma, non-Hodgkin lymphoma, or Waldenström's macroglobulinemia. Am. J. Hematol. 91, 400-405 (2016).

63. Lendvai, N. et al. Phase IB study of cabozantinib in patients with relapsed and/or refractory multiple myeloma. Blood 127, 2355-2356 (2016).

64. Leng, $Y$. et al. Phase II open-label study of recombinant circularly permuted TRAIL as a single-agent treatment for relapsed or refractory multiple myeloma. Chin. J. Cancer 35, 86 (2016).

65. Jain, $\mathrm{T}$. et al. Phase $\|$ trial of nab-paclitaxel in patients with relapsed or refractory multiple myeloma. Am. J. Hematol. 91, E504-E505 (2016).

66. Shah, J.J. et al. Phase I study of the novel investigational NEDD8-activating enzyme inhibitor pevonedistat (MLN4924) in patients with relapsed/ refractory multiple myeloma or Iymphoma. Clin. Cancer Res. 22, 34-43 (2016).

67. Yong, K. et al. Phase I study of KW-2478, a novel Hsp90 inhibitor, in patients with B-cell malignancies. Br. J. Cancer 114, 7-13 (2016).

68. Holkova, B. et al. A phase II trial of AZD6244 (Selumetinib, ARRY-142886), an oral MEK1/2 inhibitor, in relapsed/refractory multiple myeloma. Clin. Cancer Res. 22, 1067-1075 (2016).

69. Richardson, P. G. et al. Phase 1 study of marizomib in relapsed or relapsed and refractory multiple myeloma: NPI-0052-101 Part 1. Blood 127, 2693-2700 (2016).

70. Berenson, J. R. et al. CHAMPION-1: a phase 1/2 study of once-weekly carfilzomib and dexamethasone for relapsed or refractory multiple myeloma. Blood 127, 3360-3368 (2016).

71. Lesokhin, A. M. et al. Nivolumab in patients with relapsed or refractory hematologic malignancy: Preliminary results of a phase ib study. J. Clin. Oncol. 34, 2698-2704 (2016).

72. Harrison, S. J. et al. Phase I clinical trial of marizomib (NPI-0052) in patients with advanced malignancies including multiple myeloma: study NPI-0052102 final results. Clin. Cancer Res. 22, 4559-4566 (2016).

73. Ali, S. A. et al. T cells expressing an anti-B-cell maturation antigen chimeric antigen receptor cause remissions of multiple myeloma. Blood $\mathbf{1 2 8}$, 1688-1700 (2016).

74. Kumar, S. K. et al. Randomized phase 2 trial of ixazomib and dexamethasone in relapsed multiple myeloma not refractory to bortezomib. Blood $\mathbf{1 2 8}$, 2415-2422 (2016).

75. Cornell, R. F. et al. Eltanexor (KPT-8602), a second-generation selective inhibitor of nuclear export (SINE) compound, in patients with refractory multiple myeloma. Blood 130, Abstract \#3134 (2017).

76. Manasanch, E. E. et al. Interim results from the phase $1 \mathrm{a} / 1 \mathrm{~b}$ dose-finding study of CWP232291 (CWP291) in relapsed or refractory myeloma (RRMM) alone or in combination with lenalidomide and dexamethasone. Blood 130, 3091-3091 (2017)

77. Dispenzieri, A. et al. Phase I trial of systemic administration of Edmonston strain of measles virus genetically engineered to express the sodium iodide symporter in patients with recurrent or refractory multiple myeloma. Leukemia 31, 2791-2798 (2017).

78. Jung, S. H. et al. A phase I clinical study of autologous dendritic cell therapy in patients with relapsed or refractory multiple myeloma. Oncotarget $\mathbf{8}$, 41538-41548 (2017).

79. Baljevic, M. et al. Phase II study of the c-MET inhibitor tivantinib (ARQ 197) in patients with relapsed or relapsed/refractory multiple myeloma. Ann. Hematol. 96, 977-985 (2017).

80. Vogl, D. T. et al. Phase $1 / /$ study of the novel proteasome inhibitor delanzomib (CEP-18770) for relapsed and refractory multiple myeloma. Leuk. Lymphoma 58, 1872-1879 (2017).

81. Kumar, S. et al. Efficacy of venetoclax as targeted therapy for relapsed/ refractory $t(11 ; 14)$ multiple myeloma. Blood 130, 2401-2409 (2017).

82. Shah, J. J. et al. A phase 1 and 2 study of Filanesib alone and in combination with low-dose dexamethasone in relapsed/refractory multiple myeloma. Cancer 123, 4617-4630 (2017).

83. Ri, M. et al. A phase $\mathrm{I} / \mathrm{I}$ study for dose-finding, and to investigate the safety, pharmacokinetics and preliminary efficacy of NK012, an SN-38-incorporating macromolecular polymeric micelle, in patients with multiple myeloma. Intern. Med. 57, 939-946 (2018). 
84. Chen, $C$. et al. Safety and efficacy of selinexor in relapsed or refractory multiple myeloma and Waldenstrom macroglobulinemia. Blood 131, 855-863 (2018).

85. Richardson, P. G. et al. Ibrutinib alone or with dexamethasone for relapsed or relapsed and refractory multiple myeloma: phase 2 trial results. Br. J. Haematol. 180, 821-830 (2018).

86. Fouquet, G. et al. Phase I dose-escalation study of F50067, a humanized antiCXCR4 monoclonal antibody alone and in combination with lenalidomide and low-dose dexamethasone, in relapsed or refractory multiple myeloma. Oncotarget 9, 23890-23899 (2018)

87. Hou, J. et al. A phase1b dose escalation study of recombinant circularly permuted TRAIL in patients with relapsed or refractory multiple myeloma. Am. J. Clin. Oncol. 41, 1008-1014 (2018).

88. Trudel, S. et al. Targeting B-cell maturation antigen with GSK2857916 antibody-drug conjugate in relapsed or refractory multiple myeloma (BMA117159): a dose escalation and expansion phase 1 trial. Lancet Oncol. 19, 1641-1653 (2018).

89. Zhao, W.-H. et al. A phase 1, open-label study of LCAR-B38M, a chimeric antigen receptor $T$ cell therapy directed against $B$ cell maturation antigen, in patients with relapsed or refractory multiple myeloma. J. Hematol. Oncol. 11 141 (2018).

90. Richardson, P. G. et al. OP-106 HORIZON-Melflufen therapy for RRMM patients refractory to daratumumab and/or pomalidomide; updated results and clinical outcome. Blood 132 (Suppl. 1), 600 (2018).

91. Ribrag, V. et al. Phase $1 \mathrm{~b}$ trial of pembrolizumab monotherapy for relapsed/ refractory multiple myeloma: KEYNOTE-013. Br. J. Haematol. 186, e41-e44 (2019).

92. Jakubowiak, A. J. et al. Phase 1 study of selinexor plus carfilzomib and dexamethasone for the treatment of relapsed/refractory multiple myeloma. Br. J. Haematol. 186, 549-560 (2019).

93. Chari, A. et al. Oral selinexor-dexamethasone for triple-class refractory multiple myeloma. N. Engl. J. Med. 381, 727-738 (2019).

94. Bensinger, W. et al. Safety and tolerability of BION-1301 in adults with relapsed or refractory multiple myeloma. J. Clin. Oncol. 37, 8012-8012 (2019).

95. Ailawadhi, S. et al. A phase I study to assess the safety and pharmacokinetics of single-agent lorvotuzumab mertansine (IMGN901) in patients with relapsed and/or refractory CD-56-positive multiple myeloma. Clin. Lymphoma Myeloma Leuk. 19, 29-34 (2019).

96. Laubach, J. P. et al. A phase I/II Study of evofosfamide, a hypoxia-activated prodrug with or without bortezomib in subjects with relapsed/refractory multiple myeloma. Clin. Cancer Res. 25, 478-486 (2019).

97. Stewart, A. K. et al. Phase I study of the anti-FcRH5 antibody-drug conjugate DFRF4539A in relapsed or refractory multiple myeloma. Blood Cancer J. 9 $1-12$ (2019).

98. Martin, T. et al. Phase I trial of isatuximab monotherapy in the treatment of refractory multiple myeloma. Blood Cancer J. 9, 41 (2019).

99. Trudel, S. et al. Antibody-drug conjugate, GSK2857916, in relapsed/refractory multiple myeloma: an update on safety and efficacy from dose expansion phase I study.Blood Cancer J. 9, 1-10 (2019).
100. Raje, N. et al. Anti-BCMA CAR T-cell therapy bb2121 in relapsed or refractory multiple myeloma. N. Engl. J. Med. 380, 1726-1737 (2019).

101. $\mathrm{Xu}$, J. et al. Exploratory trial of a biepitopic CAR T-targeting B cell maturation antigen in relapsed/refractory multiple myeloma. Proc. Natl Acad. Sci. USA 116, 9543-9551 (2019)

102. Jagannath, $\mathrm{S}$. et al. Indatuximab ravtansine (BT062) monotherapy in patients with relapsed and/or refractory multiple myeloma. Clin. Lymphoma, Myeloma Leuk. 19, 372-380 (2019).

103. Cohen, A. D. et al. B cell maturation antigen-specific CAR T cells are clinically active in multiple myeloma. J. Clin. Investig. 129, 2210-2221 (2019).

104. Hari, P. et al. Efficacy and safety results from a phase $1 \mathrm{~b} / 2$, multicenter, openlabel study of oprozomib and dexamethasone in patients with relapsed and/ or refractory multiple myeloma. Leuk. Res. 83, 106172 (2019).

105. Ghobrial, I. M. et al. A phase Ib/ll study of oprozomib in patients with advanced multiple myeloma and Waldenstrom € macroglobulinemia. Clin. Cancer Res. 25, 4907-4916 (2019).

106. Boyle, E. M. et al. Daratumumab and dexamethasone is safe and effective for triple refractory myeloma patients: final results of the IFM 2014-04 (Etoile du Nord) trial. Br. J. Haematol. 187, 319-327 (2019).

107. Richardson, P. G. et al. Melflufen plus dexamethasone in relapsed and refractory multiple myeloma (O-12-M1): a multicentre, international, openlabel, phase 1-2 study. Lancet Haematol. 7, e395-e407 (2020).

108. Raab, M. S. et al. MOR202, a novel anti-CD38 monoclonal antibody, in patients with relapsed or refractory multiple myeloma: a first-in-human, multicentre, phase 1-2a trial. Lancet Haematol. 7, e381-e394 (2020).

109. Lonial, S. et al. Belantamab mafodotin for relapsed or refractory multiple myeloma (DREAMM-2): a two-arm, randomised, open-label, phase 2 study. Lancet Oncol. 21, 207-221 (2020).

110. Siegel, D. S. et al. Pomalidomide plus low-dose dexamethasone in relapsed refractory multiple myeloma after lenalidomide treatment failure. Br. J. Haematol. 188, 501-510 (2020).

111. Mikhael, J. et al. A dose-finding phase 2 study of single agent isatuximab (anti-CD38 mAb) in relapsed/refractory multiple myeloma. Leukemia $\mathbf{3 4}$ 3298-3309 (2020).

112. Brudno, J. N. et al. T cells genetically modified to express an anti-B-Cell maturation antigen chimeric antigen receptor cause remissions of poorprognosis relapsed multiple myeloma. J. Clin. Oncol. 36, 2267-2280 (2018).

113. Ghobrial, I. et al. Immunotherapy in multiple myeloma: accelerating on the path to the patient. Clin. Lymphoma, Myeloma Leuk. 19, 332-344 (2019).

114. Gagelmann, N., Ayuk, F., Atanackovic, D. \& Kröger, N. B cell maturation antigen-specific chimeric antigen receptor $T$ cells for relapsed or refractory multiple myeloma: a meta-analysis. Eur. J. Haematol. 104, 318-327 (2020).

115. Usmani, S. Z. et al. Clinical predictors of long-term survival in newly diagnosed transplant eligible multiple myeloma-an IMWG Research Project. Blood Cancer J. 8, 123 (2018).

116. Schwaederle, M. et al. Association of biomarker-based treatment strategies with response rates and progression-free survival in refractory malignant neoplasms a meta-Analysis. JAMA Oncol. 2, 1452-1459 (2016). 